\title{
Epithelial Mesenchymal Transition in Drug Resistance and Metastasis of Lung Cancer
}

\author{
Fariz Nurwidya, MD \\ Fumiyuki Takahashi, MD, PhD \\ Akiko Murakami, MD \\ Kazuhisa Takahashi, MD, PhD
}

Department of Respiratory Medicine, Juntendo University School of Medicine, Tokyo, Japan
Among all types of cancer, incidence of lung cancer remains the highest with regard to cancerrelated mortality. Problems contributing to recurrence of the disease include metastasis and drug resistance. Mounting evidence has demonstrated involvement of epithelial mesenchymal transition (EMT) in cancer progression. EMT is a critical mechanism ensuring tissue remodeling during morphogenesis of multicellular organisms. Therefore, understanding of the biology of this process for identification of potential EMT-targeted therapeutic strategies for the benefit cancer patients is necessary. This review describes recent evidence of EMT involvement in drug resistance and metastasis of cancers, with an emphasis on lung cancer.

Correspondence: Fariz Nurwidya, MD Department of Respiratory Medicine, Juntendo University School of Medicine, 2-1-1 Hongo, Bunkyo-ku, Tokyo 113-8421, Japan Tel: $81-80-3690-2529$ Fax: 81-3-5802-1617 E-mail: fariz@juntendo.ac.jp Received March 1, 2012 Accepted April 3, 2012

\author{
Key words \\ Epithelial-mesenchymal transition, Drug resistance, \\ Neoplasm metastasis, Lung neoplasms
}

\section{Introduction}

In 2008 , lung cancer was reported as the most commonly diagnosed cancer as well as the leading cause of cancer death worldwide in males; among females, it was the fourth most commonly diagnosed cancer and the second leading cause of cancer death [1]. When treated with the most active combination of conventional chemotherapeutic agents, the median period of survival for patients with metastatic non-small cell lung cancer (NSCLC) is eight to 10 months [2]. Failure of treatment is attributed to metastasis and recurrence [3].

Some patients exhibit intrinsic resistance to chemotherapy, while other patients, although initially sensitive to chemotherapy, eventually develop acquired resistance, even after combination therapy. Indeed, metastasis and therapeutic resistance have been reported as the major causes of failure of cancer treatment [3]. To circumvent this situation, a new class of drugs that specifically target certain molecular pathways leading to cancer phenotypes is under development [4]. Small molecules that specifically inhibit the tyrosine kinase (tyrosine kinase inhibitors [TKI]) activity of epidermal growth factor receptor (EGFR), such as gefitinib and erlotinib, were the first drugs to become clinically available for treatment of patients with NSCLC [4]. However, despite the efficacy of these drugs in patients with activating mutations, development of acquired resistance in these targeted therapies seems inevitable [5]. Results of multiple prospective trials have shown a response rate of $70 \%$ to $75 \%$ to TKIs for patients with tumors harboring EGFR mutations [6]. However, this finding indicates that another $25 \%$ to $30 \%$ of patients have intrinsic resistance to EGFRTKIs, even though their tumors harbor activating mutations in EGFR. In addition, almost without exception, even patients who show a dramatic response to initial treatment also develop acquired resistance to EGFRTKI after varying periods of time [7]. Failure of treatment may lead to metastasis of lung cancer. Among organ targets, brain metastasis is a 
frequent complication of lung cancer and is associated with significant morbidity and mortality [8].

Association of resistance to gefitinib and erlotinib in NSCLC cell lines with epithelial mesenchymal transition (EMT) has been reported [9]. Recently acquired evidence now indicates that EMT of tumor cells not only causes drug resistance, but also increases metastasis [10]. Therefore, in order to pursue possible EMT-inhibiting therapy, understanding of the biology of EMT in cancer is necessary. Findings from Pubmed analysis of EMT and cancer have demonstrated an exponential increase in the number of papers and reviews published under these terms in the last few years [11]. This review describes recent evidence of EMT involvement in drug resistance and metastasis of cancers, with an emphasis on lung cancer.

\section{EMT in Developmental State and Cancer Progression}

An epithelium is a collection of cells forming a relatively thin sheet or layer due to mutual and extensive lateral adherence of constituent cells by cell-to-cell junctions [12]. The layer is polarized, with the two sides showing non-identical properties, so that the sides can be defined as, say, inside or outside, or, more precisely, apical and basal [12].

Conversely, mesenchymal cells generally do not exhibit either regimented structure or tight intracellular adhesion [13]. Mesenchymal cells form structures that are irregular in shape, without uniformity in composition or density. Adhesions between mesenchymal cells are not as strong as those of their epithelial counterparts, which allows for increased migratory capacity [13].

The term EMT refers to a complex molecular and cellular program by which epithelial cells shed their differentiated characteristics, including cell-cell adhesion, planar and apical-basal polarity, and lack of motility, and instead acquire mesenchymal features, including motility, invasiveness, and a heightened resistance to apoptosis [14]. The EMT program plays an important role during morphogenesis of multicellular organisms. For example, during gastrulation, epithelial cells located in the primitive streak undergo EMT followed by ingression and migration to a new location where they form endodermal and mesodermal embryonic tissues [15].

However, induction of EMT can compromise the mechanical and physiological integrity of the tissue, and the consequences of inappropriate induction of this process can be disastrous. This can occur in cases of chronic inflammation, which can stimulate fibrosis, a condition in which excess EMT compromises tissue integrity and organ function, or when EMT is acquired by tumor cells [16].

\section{Classification of EMT}

Classification of EMTs into three different biological subtypes based on the biological context in which they occur has been proposed. Kalluri and Weinberg [17] proposed the term 'type 1'EMT. These EMTs, which are associated with implantation, embryo formation, and organ development, are organized for generation of diverse cell types that share common mesenchymal phenotypes. This class of EMTs does not cause fibrosis, nor does it induce an invasive phenotype resulting in systemic spread via the circulation. The type 2 EMT is associated with wound healing, tissue regeneration, and organ fibrosis [17]. In type 2 EMTs, the program begins as part of a repair-associated event that normally results in generation of fibroblasts and other related cells for reconstruction of tissues following trauma and inflammatory injury. However, in contrast to type 1 EMTs, type 2 EMTs are associated with inflammation, and cease once inflammation is attenuated, as observed during wound healing and tissue regeneration. Type 3 EMTs occur in neoplastic cells that have previously undergone genetic and epigenetic changes, specifically in genes that favor clonal outgrowth and development of localized tumors [17].

E-cadherin is a cell-cell adhesion molecule that participates in homotypic, calcium-dependent interactions for formation of epithelial adherent junctions and sequestration of $\beta$-catenin [18]. Several transcription factors have been implicated in transcriptional repression of E-cadherin. EMT inducers can be classified according to: 1) their effects on the E-cadherin promoter, and 2) source of the stimuli. Based on their effects on the E-cadherin promoter, there are two types of EMT inducer. The first type, such as Snail, ZEB, E47, and KLF8 factors, represses the activity of the E-cadherin promoter [19], and the second type, such as Twist, Goosecoid, $\mathrm{E} 2.2$, and FoxC2, is involved in indirect repression of E-cadherin transcription [19].

Regardless of the mechanism, E-cadherin repressors function as full EMT inducers in many cellular contexts, regulating expression of a variety of genes involved in repression of the epithelial character and promotion of the mesenchymal state [19]. Meanwhile, based on the source of stimuli, EMT inducers can be classified into two groups: external stimuli (that is microenvironment) and internal stimuli.

\section{External stimuli and transcriptional factors}

The tumor microenvironment, which is composed of extracellular matrix, cells, and soluble factors, plays an important role in EMT induction and further in metastasis [20]. Several stromal cell subtypes, including macrophages, contribute to tumor progression through induction of EMT at the invasive front, a mechanism also linked to metastasis [21].

Tissue-culture studies have been instrumental in defining molecular regulation of EMT. Findings from these studies have demonstrated that signalling pathways for induction of EMT have many common endpoints, including down-regulation of E-cadherin expression and expression of EMT-associated genes; in addition, triggering of EMT by several extracellular activators, and existence of extensive crosstalk between signalling 
pathways involved in activation and repression of EMT have also been reported [22].

Several tyrosine kinase receptors, including mesenchymal-epithelial transition (MET), fibroblast growth factor, insulin growth factor, epithelial growth factor family members, and, more recently, platelet derived growth factor, also play critical roles in regulation of EMT-like morphogenetic events that occur during development [23].

The three members of the Snail family, known as Snail (Snaill), Slug (Snail2), and Smuc (Snail3), encode zinc-finger type transcription factors [18]. The carboxyl-terminal domain, containing four to six C2H2-type zinc fingers, is the most conserved feature of the Snail family of proteins [18]. Evidence has indicated a fundamental role of Snail in EMT through its suppression of E-cadherin [24]. Up-regulation and nuclear accumulation of Snail have shown correlation with the EMT program in tumor tissues [25]. In addition, Kudo-Saito et al. [26] reported on acceleration of cancer metastasis by Snail-induced EMT through enhanced invasion.

The transcriptional repressor zinc-finger E-box binding homeobox 1 (ZEB1) is a crucial inducer of EMT in various human tumours, and was recently shown to promote invasion and metastasis of tumour cells [27]. ZEB1 promotes EMT through repression of genes such as E-cadherin, which is involved in maintenance of the epithelial phenotype and activation of those required for transformation to the mesenchymal phenotype [28]. Repression of multiple key determinants of epithelial differentiation and cell-cell adhesion, including the cell polarity genes Crumbs3, HUGL2, and Pals1-associated tight junction protein, by ZEB1 has also been demonstrated [29]. Results of reporter assays showed an association of ZEB1 with endogenous promoters in vivo, as well as strong repression of promoter activities [29]. Early evidence from studies conducted using mouse models, as well as a wide range of primary human carcinomas, demonstrated induction of an EMT by ZEB proteins, linking their expression with increased aggressiveness and metastasis [30].

The increase in expression of Bmi-1 was accompanied by downregulation of E-cadherin expression and up-regulation of vimentin expression in lung squamous cell carcinoma tissues [31]. Over-expression of Bmi- 1 has been reported to increase the motility and invasive properties of immortalized human mammary epithelial cells (HMLEs), which is concurrent with increased expression of mesenchymal markers, decreased expression of epithelial markers, stabilization of Snail, and dysregulation of the Akt/GSK3b pathway [32]. Silencing of endogenous Bmi-1 expression has been shown to result in reversal of EMT and reduction of motility [33]. Some data have suggested that Bmi-1 expression may be regulated by Twist1 [34].

\section{Internal stimuli}

The prominent idea is that, although some factors, such as transforming growth factor- $\beta$ (TGF- $\beta$ ), could possibly trigger EMT, accumulation of particular gene mutations would be required in order to unlock or maintain the EMT program. Indeed, gene alterations concerning extracellular receptors and/or consequent signal transduction are common in cancer [20]. Pirozzi et al. [35] reported that treatment with TGF- $\beta 1$ induced morphologic changes in NSCLC cell lines, characterized by loss of epithelial morphology assuming a fibroblast-like appearance. Treatment with TGF- $\beta 1$ has also been shown to promote a shift from epithelial to mesenchymal phenotype [35]. Induced autocrine expression of TGF- $\beta$ resulted in changes in markers and phenotype that were consistent with EMT, a modest effect on growth rate, and a shift to a more invasive phenotype [36]. In addition, several other mechanisms having the capacity to suppress E-cadherin expression, including somatic mutations, promoter hypermethylation, and histone deacetylation, have also been described [30].

\section{Role of EMT in Acquired Drug Resistance}

EMT may well play a role in determining sensitivity to EGFR-TKI. Thomson et al. [37] reported that human NSCLC lines containing wild-type EGFR, grown both in culture and as xenografts, display a range of sensitivities to EGFR inhibition, which is dependent on the degree to which they have undergone EMT. NSCLC lines expressing the epithelial cell junction protein E-cadherin showed greater sensitivity to EGFR inhibition in vitro and in xenografts. By contrast, NSCLC lines that had undergone EMT, expressing vimentin and/or fibronectin, were insensitive to the growth inhibitory effects of EGFR kinase inhibition in vitro and in xenografts.

In another study by Xie et al. [38], highly up-regulated expression of Notch-1 was observed in gefitinib-resistant PC9/AB2 lung cancer cells. Promotion of the EMT phenotype by the Notch-1 receptor intracellular domain (N1IC), the activated form of the Notch-1 receptor, was observed in PC9 cells. Silencing of Notch-1 using siRNA resulted in reversal of the EMT phenotype and restored sensitivity to gefitinib in PC9/AB2 cells. In addition, involvement of Notch-1 reduction in inhibition of anoikis, as well as colony-formation activity of PC9/AB2 cells, was also observed. Taken together, these results provide strong molecular evidence that gefitinib-acquired resistance in lung cancer cells undergoing EMT occurs through activation of Notch-1 signaling.

Using cancer specimens, Uramoto et al. [39] investigated the association between EMT status and acquired resistance. Immunohistochemical staining was performed for analysis of protein expression of epithelial and mesenchymal markers in tumour samples from patients with lung adenocarcinoma. All patients showed positive expression of epithelial markers in sensitive tumours. Findings from the study suggest that epithelial markers tended toward a slight decrease in resistance, compared with sensitive tumours. By contrast, mesenchymal markers tended toward a slight increase; however, the differences were not statistically significant [39].

However, according to findings from another study, continuous exposure of A549, a lung adenocarcinoma cell line, to gefitinib also resulted in induction of EMT [40]. Rho et al. [40] reported on use of a gefitinibresistant subline (A549/GR) derived from the parental A549 cell line 
through chronic and repeated exposure to gefitinib. Phenotypic changes, including a spindle-cell shape and increased pseudopodia formation, suggesting EMT, were observed in A549/GR cells. These changes were accompanied by a decrease of E-cadherin and an increase of vimentin, a mesenchymal marker. In addition, A549/GR cells showed 7.7-fold greater resistance to gefitinib and demonstrated cross-resistance against other EGFR-TKI, including CL-387,758, erlotinib, and ZD6478.

\section{EMT-Mediated Lung Cancer Metastasis}

Metastasis occurs when genetically unstable cancer cells adapt to a tissue microenvironment that is distant from the primary tumor [41]. Origins of invasive and metastatic phenotypes of carcinoma cells have been the subject of intensive investigation. Tumor metastasis involves a sequential series of processes that promote and regulate the escape of migratory cancer cells for generation of metastatic lesions at distant sites [42]. Mounting evidence has suggested an association of metastasis of primary epithelial tumor cells, the primary cause of death in cancer patients, with loss of E-cadherin-mediated cell-cell contacts and gain of a mesenchymal phenotype, potentially facilitating cellular motility and invasion [43].

Molecular technologies, including DNA, antibody, and proteomic arrays, and short-hairpin RNA libraries, have been used for identification of multiple molecules that contribute to the metastatic process, including growth factors, cytokines and chemokines, pro-angiogenic factors, extracellular matrix-remodeling molecules, and, most recently, transcription factors that may regulate cellular changes during tumor invasion-metastasis [44]. One view proposes that metastatic traits are acquired through exposure of epithelial cancer cells to paracrine signals received from mesenchymal cell types within the tumour-associated stroma [45]. Metastatic colonization involves lodgment or adherence of cancer cells in the microvasculature and subsequent migration of those cells across the endothelium into a secondary organ site [46].

\section{EMT Leads to Cancer Stem Cells}

Cancer stem cells have the capacity for division and expansion of the cancer stem cell pool and for differentiation into the heterogeneous non-tumorigenic cancer cell types that, in most cases, appear to constitute the bulk of cancer cells within the tumor [47].

A four-week period of exposure of immortalized human bronchial epithelial cells to tobacco carcinogens has been shown to induce a persistent, irreversible, and multifaceted dedifferentiation program marked by EMT and the emergence of stem cell-like properties [48].

Induction of EMT in immortalized HMLEs has been reported to result in acquisition of mesenchymal traits, as well as expression of stem-cell markers [49]. In addition, these cells have an increased ability to form mammospheres, a property associated with mammary epithelial stem cells [49,50]. Cancer cells in EMT, that is, EMT cells, share many properties with the classical so-called 'cancer stem cells (CSCs).' In fact, there are many indications that CSC-tumor propagating cells present characteristics of EMT cells, and, conversely, that EMT cells acquire properties of the so-called 'CSCs' (for example, markers CD44+/CD24+/-, dormancy, etc.) and that these are reversible [51].

Ding et al. [52] investigated the question of whether CSC markers are associated with EMT. For Capan1M9, a highly migratory cell subclone established from human pancreatic cancer cell line Capan-1, expression, migration, and invasion of $\mathrm{CD} 133$ were greater than that for the parent cells. Up-regulation of EMT-related transcription factors Slug and Snail, as well as a substantial increase in $\mathrm{N}$-cadherin and fibronectin, were observed in Capan1M9 cells. By contrast, occludin and desmoplakin were suppressed. Knockdown of endogenous CD133 in Capan1M9 cells led to suppression of Slug and reduction of migration and invasion. Taken together, CD133 has an important role in migration and invasion through facilitation of EMT in pancreatic cancer cells.

On the basis of their studies, Brabletz et al. [53] postulated that EMT and stem-cell properties are combined in invasive cancer cells-which often co-express EMT and stem-cell markers-and proposed the concept of the 'migrating cancer stem cell.' This concept links two features: 1) classical EMT-associated features that support tumor cell motility and dissemination, and allow for survival of cells under the stressful conditions of a new environment; and 2) the associated stem-cell properties, including prevention of senescence, allowing for unlimited reconstitution of the tumor at a distant site, leading ultimately to metastasis [53].

\section{Clinical Prognostic of EMT and Current EMT-Targeted Therapy}

Soltermann et al. [54] investigated the prognostic significance of the EMT indicator proteins periostin and vimentin in comparison with versican, a putative indicator of the opposite mechanism, MET, and the desmoplasia proteins collagen and elastin in NSCLC. Tumors of 533 patients with surgically resected NSCLC were used for analysis of stromal and epithelial protein expression by immunohistochemistry. High expression of versican in either stroma or epithelia as well as of stromal collagen showed fewer but concordant associations with advanced tumor and periostin, respectively. High expression of elastin showed an opposite association with less advanced disease.

Loss of epithelial proteins and/or acquisition of mesenchymal proteins are associated with poorly differentiated histology, advanced stage, and poor outcome [55]. However, findings from the study also revealed that EMT status in the primary tumor is not predictive of postoperative recurrence or disease-free survival in patients with lung cancer [56].

EMT has been established as a mechanism that confers tumor cells 
with abilities essential for drug resistance, metastasis, and acquired-tumor stem cell traits. Therefore, inhibition of EMT can be a critical therapeutic strategy for prevention of tumor progression. Forced expression of miR200 resulted in abrogation of the capacity of lung cancer cell lines to undergo EMT, invade, and metastasize, and conferred transcriptional features of metastasis-incompetent tumor cells [57]. Restoration of E-cadherin-mediated cell adhesion is another means by which prevention of EMT in cancer might be possible [58]. Inhibition of EMT by NPI-0052, a protesome inhibitor, via inhibition of nuclear factor- $\mathrm{\kappa} \mathrm{B}$ and Snail has also been demonstrated [59], whereas over-expression of FOXA2 resulted in reduced invasion and suppression of TGF- $\beta 1$-induced EMT [60]. Meanwhile, activation of peroxisome proliferator-activated receptor-g was reported to inhibit TGF- $\beta$-induced EMT in lung cancer cells and prevent metastasis by antagonizing $\operatorname{Smad} 3$ function [61].

\section{Conclusion}

Research on the role of EMT in cancer progression, in terms of drug resistance and metastasis, is currently in a very active phase, with several significant publications every year. At this pace, it seems likely that we will soon have a better understanding of how to inhibit this crucial process in order to prevent cancer progression.

Given the role of EMT in promoting chemoresistance, invasion, and stem cell-like properties, specific targeting of EMT could potentially serve to decrease metastasis and overcome drug resistance; however, significant additional work is needed in order to translate these findings into meaningful therapies.

\section{Conflicts of Interest}

Conflict of interest relevant to this article was not reported.

\section{References}

1. Jemal A, Bray F, Center MM, Ferlay J, Ward E, Forman D. Global cancer statistics. CA Cancer J Clin. 2011;61:69-90.

2. Ohe Y, Ohashi Y, Kubota K, Tamura T, Nakagawa K, Negoro S, et al. Randomized phase III study of cisplatin plus irinotecan versus carboplatin plus paclitaxel, cisplatin plus gemcitabine, and cisplatin plus vinorelbine for advanced non-small-cell lung cancer: Four-Arm Cooperative Study in Japan. Ann Oncol. 2007;18:317-23.

3. Xiao D, He J. Epithelial mesenchymal transition and lung cancer. J Thorac Dis. 2010;2:1549.

4. Mitsudomi T. Advances in target therapy for lung cancer. Jpn J Clin Oncol. 2010;40:101-6. 5. Giaccone G. EGFR point mutation confers resistance to gefitinib in a patient with non-smallcell lung cancer. Nat Clin Pract Oncol. 2005;2:296-7.

6. Mitsudomi T, Yatabe Y. Mutations of the epidermal growth factor receptor gene and related genes as determinants of epidermal growth factor receptor tyrosine kinase inhibitors sensitivity in lung cancer. Cancer Sci. 2007:98:1817-24.

7. Yano S, Wang W, Li Q, Matsumoto K, Sakurama H, Nakamura T, et al. Hepatocyte growth factor induces gefitinib resistance of lung adenocarcinoma with epidermal growth factor receptor-activating mutations. Cancer Res. 2008:68:9479-87.

8. Jamal-Hanjani M, Spicer J. Epidermal growth factor receptor tyrosine kinase inhibitors in the treatment of epidermal growth factor receptor-mutant non-small cell lung cancer metastatic to the brain. Clin Cancer Res. 2012;18:938-44.

9. Yauch RL, Januario T, Eberhard DA, Cavet G, Zhu W, Fu L, et al. Epithelial versus mesenchymal phenotype determines in vitro sensitivity and predicts clinical activity of erlotinib in lung cancer patients. Clin Cancer Res. 2005;11(24 Pt 1):8686-98.

10. Singh A, Settleman J. EMT, cancer stem cells and drug resistance: an emerging axis of evil in the war on cancer. Oncogene. 2010;29:4741-51.

11. Thompson EW, Williams ED. EMT and MET in carcinoma: clinical observations, regulatory pathways and new models. Clin Exp Metastasis. 2008;25:591-2.

12. Thompson EW, Newgreen DF, Tarin D. Carcinoma invasion and metastasis: a role for epithelial-mesenchymal transition? Cancer Res. 2005;65:5991-5.

13. Lee JM, Dedhar S, Kalluri R, Thompson EW. The epithelial-mesenchymal transition: new insights in signaling, development, and disease. J Cell Biol. 2006;172:973-81.

14. Polyak K, Weinberg RA. Transitions between epithelial and mesenchymal states: acquisition of malignant and stem cell traits. Nat Rev Cancer. 2009;9:265-73.

15. Battula VL, Evans KW, Hollier BG, Shi Y, Marini FC, Ayyanan A, et al. Epithelial-mesenchymal transition-derived cells exhibit multilineage differentiation potential similar to mesenchymal stem cells. Stem Cells. 2010;28:1435-45.
16. Radisky DC. Epithelial-mesenchymal transition. J Cell Sci. 2005;118(Pt 19):4325-6.

17. Kalluri R, Weinberg RA. The basics of epithelial-mesenchymal transition. J Clin Invest. 2009:119:1420-8.

18. Shih JY, Yang PC. The EMT regulator slug and lung carcinogenesis. Carcinogenesis. 2011;32:1299-304

19. Thiery JP, Acloque H, Huang RY, Nieto MA. Epithelial-mesenchymal transitions in development and disease. Cell. 2009;139:871-90.

20. Voulgari A, Pintzas A. Epithelial-mesenchymal transition in cancer metastasis: mechanisms, markers and strategies to overcome drug resistance in the clinic. Biochim Biophys Acta. 2009:1796:75-90.

21. Bonde AK, Tischler V, Kumar S, Soltermann A, Schwendener RA. Intratumoral macrophages contribute to epithelial-mesenchymal transition in solid tumors. BMC Cancer. 2012;12:35.

22. Thiery JP, Sleeman JP. Complex networks orchestrate epithelial-mesenchymal transitions. Nat Rev Mol Cell Biol. 2006;7:131-42.

23. Yang J, Weinberg RA. Epithelial-mesenchymal transition: at the crossroads of development and tumor metastasis. Dev Cell. 2008;14:818-29.

24. Zhou BP, Deng J, Xia W, Xu J, Li YM, Gunduz M, et al. Dual regulation of Snail by GSK3beta-mediated phosphorylation in control of epithelial-mesenchymal transition. Nat Cell Biol. 2004;6:931-40.

25. Lee MY, Chou CY, Tang MJ, Shen MR. Epithelial-mesenchymal transition in cervical cancer: correlation with tumor progression, epidermal growth factor receptor overexpression, and snail up-regulation. Clin Cancer Res. 2008;14:4743-50.

26. Kudo-Saito C, Shirako H, Takeuchi T, Kawakami Y. Cancer metastasis is accelerated through immunosuppression during Snail-induced EMT of cancer cells. Cancer Cell. 2009;15:195206.

27. Burk U, Schubert J, Wellner U, Schmalhofer O, Vincan E, Spaderna S, et al. A reciprocal repression between ZEB1 and members of the miR-200 family promotes EMT and invasion in cancer cells. EMBO Rep. 2008;9:582-9.

28. Hurt EM, Saykally JN, Anose BM, Kalli KR, Sanders MM. Expression of the ZEB1 (deltaEF1) transcription factor in human: additional insights. Mol Cell Biochem. 2008;318:89-99.

29. Aigner K, Dampier B, Descovich L, Mikula M, Sultan A, Schreiber M, et al. The transcription factor ZEB1 (deltaEF1) promotes tumour cell dedifferentiation by repressing master regulators of epithelial polarity. Oncogene. 2007;26:6979-88.

30. Dasari V, Gallup M, Lemjabbar H, Maltseva I, McNamara N. Epithelial-mesenchymal transition in lung cancer: is tobacco the "smoking gun"? Am J Respir Cell Mol Biol. 2006;35:3-9.

31. Huang J, Qiu Y, Chen G, Huang L, He J. The relationship between Bmi-1 and the epithelial- 
mesenchymal transition in lung squamous cell carcinoma. Med Oncol. 2012;29:1606-13

32. Guo BH, Feng Y, Zhang R, Xu LH, Li MZ, Kung HF, et al. Bmi-1 promotes invasion and metastasis, and its elevated expression is correlated with an advanced stage of breast cancer. Mol Cancer. 2011:10:10.

33. Song LB, Li J, Liao WT, Feng Y, Yu CP, Hu LJ, et al. The polycomb group protein Bmi-1 represses the tumor suppressor PTEN and induces epithelial-mesenchymal transition in human nasopharyngeal epithelial cells. J Clin Invest. 2009;119:3626-36.

34. Yang MH, Hsu DS, Wang HW, Wang HJ, Lan HY, Yang WH, et al. Bmi1 is essential in Twist1induced epithelial-mesenchymal transition. Nat Cell Biol. 2010;12:982-92.

35. Pirozzi G, Tirino V, Camerlingo R, Franco R, La Rocca A, Liguori E, et al. Epithelial to mesenchymal transition by TGFbeta-1 induction increases stemness characteristics in primary non small cell lung cancer cell line. PLoS One. 2011;6:e21548.

36. Argast GM, Krueger JS, Thomson S, Sujka-Kwok I, Carey K, Silva S, et al. Inducible expression of TGFbeta, snail and Zeb1 recapitulates EMT in vitro and in vivo in a NSCLC model. Clin Exp Metastasis. 2011:28:593-614.

37. Thomson S, BuckE, Petti F, Griffin G, Brown E, Ramnarine N, et al. Epithelial to mesenchymal transition is a determinant of sensitivity of non-small-cell lung carcinoma cell lines and xenografts to epidermal growth factor receptor inhibition. Cancer Res. 2005;65:9455-62.

38. Xie M, Zhang L, He CS, Xu F, Liu JL, Hu ZH, et al. Activation of Notch-1 enhances epithelialmesenchymal transition in gefitinib-acquired resistant lung cancer cells. J Cell Biochem. 2012;113:1501-13.

39. Uramoto H, Iwata T, Onitsuka T, Shimokawa H, Hanagiri T, Oyama T. Epithelial-mesenchymal transition in EGFR-TKI acquired resistant lung adenocarcinoma. Anticancer Res. 2010; 30:2513-7.

40. Rho JK, Choi YJ, Lee JK, Ryoo BY, Na, II, Yang SH, etal. Epithelial to mesenchymal transition derived from repeated exposure to gefitinib determines the sensitivity to EGFR inhibitors in A549, a non-small cell lung cancer cell line. Lung Cancer. 2009;63:219-26.

41. Gupta GP, Massague J. Cancer metastasis: building a framework. Cell. 2006;127:679-95

42. Barr S, Thomson S, Buck E, Russo S, Petti F, Sujka-Kwok I, et al. Bypassing cellular EGF receptor dependence through epithelial-to-mesenchymal-like transitions. Clin Exp Metastasis. 2008:25:685-93

43. Eastham AM, Spencer H, Soncin F, Ritson S, Merry CL, Stern PL, et al. Epithelial-mesenchymal transition events during human embryonic stem cell differentiation. Cancer Res. 2007; 67:11254-62.

44. Talmadge JE, Fidler IJ. AACR centennial series: the biology of cancer metastasis: historical perspective. Cancer Res. 2010;70:5649-69.

45. Karnoub AE, Dash AB, Vo AP, Sullivan A, Brooks MW, Bell GW, et al. Mesenchymal stem cells within tumour stroma promote breast cancer metastasis. Nature. 2007;449:557-63.

46. Drake JM, Strohbehn G, Bair TB, Moreland JG, Henry MD. ZEB1 enhances transendothelial migration and represses the epithelial phenotype of prostate cancer cells. Mol Biol Cell. 2009:20:2207-17.

47. Clarke MF, Dick JE, Dirks PB, Eaves CJ, Jamieson CH, Jones DL, et al. Cancer stem cells: perspectives on current status and future directions: AACR Workshop on cancer stem cells. Cancer Res. 2006;66:9339-44.

48. Tellez CS, Juri DE, Do K, Bernauer AM, Thomas CL, Damiani LA, et al. EMT and stem cell-like properties associated with miR-205 and miR-200 epigenetic silencing are early manifestations during carcinogen-induced transformation of human lung epithelial cells. Cancer Res. 2011;71:3087-97.

49. Mani SA, Guo W, Liao MJ, Eaton EN, Ayyanan A, Zhou AY, et al. The epithelial-mesenchymal transition generates cells with properties of stem cells. Cell. 2008;133:704-15.

50. Morel AP, Lievre M, Thomas C, Hinkal G, Ansieau S, Puisieux A. Generation of breast cancer stem cells through epithelial-mesenchymal transition. PLoS One. 2008;3:e2888.

51. Floor S, van Staveren WC, Larsimont D, Dumont JE, Maenhaut C. Cancer cells in epithelialto-mesenchymal transition and tumor-propagating-cancer stem cells: distinct, overlapping or same populations. Oncogene. 2011;30:4609-21.

52. Ding Q, Yoshimitsu M, Kuwahata T, Maeda K, Hayashi T, Obara T, et al. Establishment of a highly migratory subclone reveals that CD133 contributes to migration and invasion through epithelial-mesenchymal transition in pancreatic cancer. Hum Cell. 2012;25:1-8.

53. Brabletz S, Brabletz T. The ZEB/miR-200 feedback loop: a motor of cellular plasticity in development and cancer? EMBO Rep. 2010;11:670-7.

54. Soltermann A, Tischler V, Arbogast S, Braun J, Probst-Hensch N, Weder W, et al. Prognostic significance of epithelial-mesenchymal and mesenchymal-epithelial transition protein expression in non-small cell lung cancer. Clin Cancer Res. 2008;14:7430-7.

55. Kim MA, Lee HS, Lee HE, Kim JH, Yang HK, Kim WH. Prognostic importance of epithelialmesenchymal transition-related protein expression in gastric carcinoma. Histopathology. 2009;54:442-51

56. Chikaishi Y, Uramoto H, Tanaka F. The EMT status in the primary tumor does not predict postoperative recurrence or disease-free survival in lung adenocarcinoma. Anticancer Res. 2011;31:4451-6.

57. Gibbons DL, Lin W, Creighton CJ, Rizvi ZH, Gregory PA, Goodall GJ, et al. Contextual extracellular cues promote tumor cell EMT and metastasis by regulating miR-200 family expression. Genes Dev. 2009;23:2140-51.

58. Thiery JP. Epithelial-mesenchymal transitions in tumour progression. Nat Rev Cancer. 2002;2:442-54.

59. Baritaki S, Chapman A, Yeung K, Spandidos DA, Palladino M, Bonavida B. Inhibition of epithelial to mesenchymal transition in metastatic prostate cancer cells by the novel proteasome inhibitor, NPI-0052: pivotal roles of Snail repression and RKIP induction. Oncogene. 2009:28:3573-85.

60. Tang Y, Shu G, Yuan X, Jing N, Song J. FOXA2 functions as a suppressor of tumor metastasis by inhibition of epithelial-to-mesenchymal transition in human lung cancers. Cell Res. 2011;21:316-26

61. Reka AK, Kurapati H, Narala VR, Bommer G, Chen J, Standiford TJ, et al. Peroxisome proliferator-activated receptor-gamma activation inhibits tumor metastasis by antagonizing Smad3mediated epithelial-mesenchymal transition. Mol Cancer Ther. 2010;9:3221-32. 